\title{
Accelerated, Pulsed Collagen Cross-Linking versus the Dresden Protocol in Keratoconus: A Case Series
}

\author{
Nikolaos Dervenis ${ }^{\mathrm{a}, \mathrm{b}}$ Panagiotis Dervenis ${ }^{\mathrm{a}, \mathrm{c}}$ Nikolaos Dragoumis $^{\mathrm{a}}$ \\ Andreas Papandroudis ${ }^{a}$ Zachos Zachariadis $^{\mathrm{a}}$ Miltos Balidis $^{\mathrm{a}}$ \\ a Ophthalmology Unit, Ophthalmica Eye Institute, Thessaloniki, Greece; boorfields Eye Hospital, London, UK; \\ 'Department of Ophthalmology, General Hospital of Trikala, Trikala, Greece
}

\section{Significance of the Study}

- Corneal stroma demarcation line depth did not differ significantly between the eyes treated with the pulsed, accelerated collagen cross-linking (CXL) protocol and the Dresden protocol. This supports the suggestion that the two techniques may have similar efficacies in the treatment of keratoconus.

- The use of pulsed light compared to continuous light may improve the availability of oxygen during CXL treatment, resulting in better postoperative outcomes.

\section{Keywords}

Cornea $\cdot$ Ectasia $\cdot$ Epithelium-off collagen cross-linking · Riboflavin

\begin{abstract}
Purpose: The aim of our study was to compare the depth of the demarcation line developing in the cornea after the standard Dresden protocol versus the accelerated, pulsed, epithelium-off corneal collagen cross-linking (CXL). Methods: This was a nonrandomized, retrospective case series. Patients with progressive keratoconus were treated with either the standard Dresden protocol (Group 1) or accelerated, epithelium-off CXL using the Avedro (Waltham, MA, USA) device (Group 2). The accelerated CXL protocol involved 18 min of pulsed ultraviolet- $A\left(20 \mathrm{~mW} / \mathrm{cm}^{2}, 7.2 \mathrm{~J} / \mathrm{cm}^{2}\right.$, pulsed pro-
\end{abstract}

file: $1 \mathrm{~s}$ on, $2 \mathrm{~s}$ off). The depth of the demarcation line was measured about 3 months postoperatively. Results: Fiftynine eyes of 35 subjects were included in the analysis. Group 1 consisted of 19 eyes, and Group 2 of 40 eyes. The mean age of the participants was 22.21 years in Group 1 and 26.55 years in Group 2 ( $p=0.184$ ). The mean preoperative $\mathrm{K}$ value was 44.89 D in Group 1 and 45.20 D in Group 2 ( $p=0.768$ ). The depth of the demarcation line was $322.50 \mu \mathrm{m}$ in Group 1 and $319.95 \mu \mathrm{m}$ in Group 2 ( $p=0.937)$. Conclusions: The demarcation line depth was not statistically significantly different between the two protocols. The significance of the demarcation line depth has not been fully clarified in the literature. Our results support the contention that these two techniques may have similar structural outcomes and efficacies in the treatment of keratoconus.

(C) 2020 The Author(s)

Published by S. Karger AG, Basel

Nikolaos Dervenis

Ophthalmology Unit

Ophthalmica Eye Institute

GR-546 55 Thessaloniki (Greece)

nikosdervenis@gmail.com 


\section{Introduction}

Keratoconus (KC) is a bilateral, noninflammatory corneal ectasia, associated with gradual loss of visual acuity (VA). It is characterized by progressive, noninflammatory, inferior corneal thinning, steepening, and apical scarring [1].

Several treatment methods can be offered to patients with KC. These include refractive correction (with glasses or contact lenses), implantation of intracorneal ring segments, and, in advanced stages, corneal transplantation (penetrating keratoplasty or deep anterior lamellar keratoplasty) $[2,3]$.

In contrast to other methods, corneal collagen crosslinking (CXL) can provide stiffening of the cornea and possibly alter the natural history of KC. It is a well-established method and can reduce the risk of $\mathrm{KC}$ progression in the long term $[4,5]$. It has been approved by the FDA recently for the treatment of $\mathrm{KC}$ and cornea ectasia. The Dresden protocol, which was the CXL technique initially described, involves $30 \mathrm{~min}$ of ultraviolet-A (UVA) irradiation at an irradiance of $3.0 \mathrm{~mW} / \mathrm{cm}^{2}$, resulting in a total surface dose of $5.4 \mathrm{~J} / \mathrm{cm}^{2}$ [6]. Based on the BunsenRoscoe law of reciprocity, the same photochemical effect can be achieved with a reduced illumination time and a correspondingly increased irradiation intensity [7]. Several new commercially available CXL devices offer higher UVA irradiances, which allow shorter treatment times with similar energy according to the Bunsen-Roscoe law.

Recently, pulsed CXL has been proposed as an alternative to the classic Dresden protocol, considering that it may increase oxygen delivery to the cornea during treatment. More specifically, during the first $10-15 \mathrm{~s}$ of a continuous UVA illumination of a riboflavin-soaked cornea (under aerobic conditions), sensitized photooxidation of the substrate occurs mainly by its reaction with photochemically generated reactive oxygen species, such as singlet molecular oxygen. This is consistent with a type II photochemical mechanism. After the first 10-15 s, when oxygen becomes totally depleted, the reaction between the substrate and riboflavin becomes consistent with a type I photochemical mechanism, which takes place under anaerobic conditions. In the type I photochemical mechanism, excited riboflavin is used as substrate that generates oxygen-free radicals by riboflavin photolysis. Consequently, pulsing UVA radiation can achieve higher oxygen concentrations by reducing oxygen consumption and restarting the type II photochemical mechanism [8].

Another recent development is the combination of CXL with refractive laser procedures in eyes with $\mathrm{KC}$.
Promising functional and structural outcomes have been reported using these techniques $[9,10]$.

A corneal stroma demarcation line has been described to develop after CXL with the Dresden protocol. It can be visualized during slit-lamp biomicroscopy 2 weeks after treatment [11]. It can also be visualized with confocal microscopy (IVCM) and anterior segment optical coherence tomography (OCT). This is represented by a hyperreflective line within the corneal stroma at approximately $300 \mu \mathrm{m}$. The depth of the demarcation line has been suggested to represent the effectiveness of CXL treatment and marks the border of the corneal stroma that is denuded from keratocytes $[12,13]$. However, some recent studies argue against this suggestion $[14,15]$.

The purpose of this study was to compare the depth of the corneal stroma demarcation line developing after the Dresden protocol and the accelerated protocol, using anterior segment OCT. Refractive laser ablation was used in some of our patients as indicated, according to the surgeon's preference.

\section{Subjects and Methods}

This is a retrospective case series analysis of patients undergoing CXL treatment, in the Ophthalmology Unit of the Ophthalmica Eye Institute, Thessaloniki, Greece. Only patients with progressive $\mathrm{KC}$ were included in this study.

KC was diagnosed based on typical corneal topography - Pentacam Scheimpflug Imaging System (Oculus, Inc.) - findings and clinical signs (Fleischer ring, Vogt striae, stromal thinning, and conical protrusion). Only Grade I and II eyes according to the Amsler-Krumeich classification were included in the study.

The inclusion criteria were progressive $\mathrm{KC}$, corneal thickness $>400 \mu \mathrm{m}$, and age $>18$ years. Exclusion criteria were age $<18$ or $>45$ years, corneal thickness $<400 \mu \mathrm{m}$, no clear demarcation line over repeated OCT scans, presence of any other corneal or anterior-segment pathology, and history of pregnancy or lactation.

For all study participants, demographic data including age and gender were recorded. Every patient underwent complete ophthalmic examination preoperatively, including corrected VA measurement. VA measurement and the ophthalmic examination were repeated at least 3 months postoperatively. All patients underwent Pentacam imaging preoperatively and at least 3 months postoperatively. Based on the Pentacam measurements, the preoperative and postoperative $\mathrm{K}$ values (minimum, maximum, and mean), as well as the preoperative and postoperative minimum corneal thickness were recorded.

Two surgeons (M.B. and Z.Z.) completed all procedures under sterile conditions using epithelial-off CXL. Topical anesthesia with proxymetacaine hydrochloride $0.5 \%$ (Alcaine) was used for all patients preoperatively. Following that, the corneal epithelium was removed using the excimer laser EX500 (Wavelight Laser Technologie AG). According to the surgeon's preference, topographyguided excimer laser stromal ablation could be performed. Twen- 
ty-one eyes received excimer ablation in combination with CXL. Previous ablation was not associated with the depth of the demarcation line $(p=0.375)$. This may be related to the fact that CXL treatment followed the application of excimer laser; therefore, CXL only affected the remaining corneal stromal bed. CXL was completed using the Avedro device (Avedro, Waltham, MA, USA). Before CXL, 0.1\% riboflavin, saline, HPMC drops (VibeX Rapid; Avedro, Waltham, MA, USA) were applied for $10 \mathrm{~min}$ (instilled on the center of the cornea every $2 \mathrm{~min}$ ). The two CXL techniques applied were the Dresden protocol involving $30 \mathrm{~min}$ of UVA irradiation $\left(370 \mathrm{~nm}, 3 \mathrm{~mW} / \mathrm{cm}^{2}\right)$ as originally described by Wollensak et al. [6] versus $18 \mathrm{~min}$ of pulsed UVA in Group $2\left(20 \mathrm{~mW} / \mathrm{cm}^{2}, 7.2\right.$ $\mathrm{J} / \mathrm{cm}^{2}$, pulsed profile: $1 \mathrm{~s}$ on, $2 \mathrm{~s}$ off). The Dresden protocol was the technique implemented first in our clinic before we switched to the newer accelerated protocol. Therefore, no systemic randomization was used but consecutive patients were enrolled in both groups.

The Spectralis (Heidelberg Engineering, Heidelberg, Germany) anterior segment spectral domain OCT was used for the measurement of the depth of the demarcation line 3 months postoperatively for all study participants. One grader (M.B.) made all measurements centrally in the cornea using the Spectralis software. Only patients with a clearly visible demarcation line were included in this study. Four patients ( 1 from Group 1 and 3 from Group 2) were excluded as the demarcation line could not be identified clearly postoperatively. We could not identify any qualitative association between the presence of the demarcation line and the treatment protocol used.

Statistical analysis was performed using SPSS for windows (version 24.0; SPSS, Chicago, IL, USA). We calculated that for an alpha value of 0.05 and a power level of $90 \%$, a sample of just over 50 subjects (1:2 enrollment ratio) would be sufficient. Independent samples $t$ test was used to compare the 2 groups, and $p$ values $<0.05$ were considered statistically significant.

\section{Results}

Fifty-nine eyes of 35 subjects were included in the analysis. Group 1 consisted of 19 (32.2\%) eyes - 11 eyes (57.9\%) had Grade I KC, and 8 eyes (42.1\%) had Grade II $\mathrm{KC}$ - and Group 2 was composed of 40 (67.8\%) eyes - 23 eyes (57.5\%) had Grade I KC, and 17 eyes (42.5\%) had Grade II KC. The descriptive statistics of the study participants and the preoperative $\mathrm{K}$ values and minimum corneal thickness are summarized in Table 1 . The mean age of the participants in Group 1 was 22.21 years, and in Group 2, it was 26.55 years $(p=0.184)$. The mean preoperative $\mathrm{K}$ value in Group 1 was 44.89 and $45.20 \mathrm{D}$ in Group $2(p=0.768)$. Preoperatively, the mean minimum corneal thickness was 413.43 and $488.20 \mu \mathrm{m}$ in Groups 1 and 2, respectively. The decreased minimum corneal thickness observed in the Dresden group might have been related to the fact that a limited range of clinical criteria were initially applied for CXL before it became a wellestablished treatment for KC. When the Dresden protocol became first available for the treatment of $\mathrm{KC}$, most
Table 1. Descriptive statistics of the study participants and preoperative $\mathrm{K}$ values, minimum corneal thickness, and VA

\begin{tabular}{lccc}
\hline Variable & Group 1 & Group 2 & $p$ value \\
\hline Age, years & 22.21 & 26.55 & 0.184 \\
Preop Kmin, D & 43.50 & 43.97 & 0.659 \\
Preop Kmax, D & 46.39 & 46.57 & 0.875 \\
Preop Kmean, D & 44.89 & 45.20 & 0.768 \\
Preop min corneal & & & \\
$\quad$ thickness, $\mu \mathrm{m}$ & 413.43 & 488.20 & $<0.001$ \\
Preop VA & 0.68 & 0.93 & 0.138 \\
KC grading, $n(\%)$ & & & \\
$\quad$ I & $11(57.9)$ & $23(57.5)$ & \\
$\quad$ II & $-8(42.1)$ & $-17(42.5)$ & \\
Gender, $n(\%)$ & & & \\
$\quad$ Male & $13(68.4)$ & $30(75)$ & \\
$\quad$ Female & $6(31.6)$ & $-10(25)$ & \\
\hline
\end{tabular}

Table 2. Postoperative K values, minimum corneal thickness, VA, and demarcation line depth in the study groups

\begin{tabular}{lccc}
\hline Variable & Group 1 & Group 2 & $p$ value \\
\hline Postop Kmin, D & 42.95 & 43.19 & 0.850 \\
Postop Kmax, D & 46.67 & 45.49 & 0.359 \\
Postop Kmean, D & 44.77 & 44.28 & 0.653 \\
Postop min corneal thickness, $\mu \mathrm{m}$ & 405.05 & 457.12 & 0.024 \\
Postop VA & 0.74 & 0.90 & 0.065 \\
Demarcation line depth, $\mu \mathrm{m}$ & 322.50 & 319.95 & 0.937 \\
Follow-up time, months & 7.2 & 6.9 & 0.570 \\
\hline
\end{tabular}

clinicians would be more conservative, considering it an option for more advanced cases of the disease, probably even as a last option before corneal transplants were taken into account. While clinical and research data confirmed the safety and efficacy of this new treatment, the criteria for its use became wider, and most clinicians would consider it an option earlier during the course of the disease. Furthermore, in several of our patients, the eye presenting with more advanced disease was treated first with the Dresden protocol, while the second eye was treated with the accelerated protocol when it became available.

The postoperative $\mathrm{K}$ values, minimum corneal thickness, and depth of demarcation line are summarized in Table 2. The depth of the demarcation line in Group 1 was 322.50 and $319.95 \mu \mathrm{m}$ in Group $2(p=0.937)$. Minimum corneal thickness was again lower in Group $1(p=0.027)$. The mean follow-up time in Group 1 was 7.2 months, and for Group 2, it was 6.9 months $(p=0.57)$. 


\section{Discussion}

The promising prospect of using accelerated CXL was developed to achieve a shorter treatment duration by increasing the illumination intensity accordingly. Its use is based on the Bunsen-Roscoe law of reciprocity, and a constant radiant exposure was maintained during the treatment. The same energy dose of $5.4 \mathrm{~J} / \mathrm{cm}^{2}$ can be delivered to stromal collagen adjusting UVA power and exposure time. A safety profile of high-fluence CXL at 18 $\mathrm{mW} / \mathrm{cm}^{2}$ for $5 \mathrm{~min}$ in vivo has been introduced [16].

Studies comparing conventional and $30 \mathrm{~mW} / \mathrm{cm}^{2}$ $\mathrm{CXL}$ and $30 \mathrm{~mW} / \mathrm{cm}^{2}$ accelerated CXL suggest that both treatment protocols demonstrate similar functional improvements. However, topography outcomes were better with conventional CXL compared with this specific accelerated CXL, which is currently abandoned by most of the surgeons [17]. A review comparing the conventional and the accelerated protocol did not show any significant differences between the treatment options as efficacy, $\mathrm{VA}$, and safety were not different between the groups [18]. A study comparing 4 irradiation protocols $(3,9,18$, and $30 \mathrm{~mW} / \mathrm{cm}^{2}$ ) at 1 year of follow-up suggested that the greatest flattening effect was for the conventional method [19]. Irradiance of $9 \mathrm{~mW} / \mathrm{cm}^{2}$ accelerated CXL has been shown to stabilize KC and corneal ectasia, improving the corneal topography and best-corrected VA at the same time. The results of the 24 months follow-up were comparable with the conventional $3 \mathrm{~mW} / \mathrm{cm}^{2} \mathrm{CXL}$. On the other hand, $18 \mathrm{~mW} / \mathrm{cm}^{2}$ accelerated CXL was less successful in flattening the cornea topography compared to the conventional CXL $[20,21]$. In a different study, $40-45 \mathrm{~mW} / \mathrm{cm}^{2}$ for $2 \mathrm{~min}$ was associated with increased corneal stiffness compared to the Dresden protocol, but this was not confirmed for intensities $>50 \mathrm{~mW} / \mathrm{cm}^{2}$. Reduced depth of the demarcation line and reduced biomechanical stiffness have been reported with these accelerated CXL techniques [22], and insufficient oxygen diffusion has been proposed as the mechanism responsible [23]. However, multiple studies have shown no significant difference between conventional and accelerated CXL regarding the uncorrected, best-corrected VA and the refractive outcome after treatment. Acceptable efficacy of accelerated protocols has been shown in all these studies $[24,25]$. By the same token, early data on the use of pulsed, accelerated CXL show that the technique is safe and effective in the treatment of KC $[26,27]$. Analysis of different pulsed profiles can help clarify the structural outcomes of CXL and assess the most effective treatment protocol.

Accelerated, Pulsed CXL versus the

Dresden Protocol in Keratoconus
On the other hand, in vivo IVCM showed that keratocyte apoptosis and treatment penetration differed significantly between continuous and pulsed, high-irradiance, UVA light, accelerated protocols. This difference may be affecting the long-term results of CXL treatment in arresting KC progression [28]. There are also concerns whether the depth of the demarcation line is predictive of the long-term outcomes of CXL [15].

Mazzotta et al. [14] conducted a trial utilizing $30 \mathrm{~mW} /$ $\mathrm{cm}^{2}$ with a higher energy dose of $7.2 \mathrm{~J} / \mathrm{cm}^{2}$ continuous and pulsed light. Visual function and refractive improvement of the accelerated CXL method were comparable with the Dresden protocol after 12 months of follow-up. However, a mean treatment penetration of about $150 \mu \mathrm{m}$ was documented after $30 \mathrm{~mW} / \mathrm{cm}^{2}$ of continuous light. IVCM and OCT imaging confirmed the presence of cellular changes including apoptosis, nerve disappearance, and demarcation line depth induced with this technique. On the contrary, penetration was deeper (average of about $220 \mu \mathrm{m}$, range 200-250) after the pulsed, accelerated CXL. Accelerated CXL with higher UVA power and reduced treatment exposure time showed less penetration, while pulsed UVA light enabled a deeper penetration. Deeper cell viability can also be achieved with prolonged exposure, while the energy dose delivered to the corneal tissue is kept stable [29].

The chemical reactions taking place during these two methods have been described recently. Riboflavin consumption in the corneal stroma has been shown to be energy dependent in accordance with the Bunsen-Roscoe law, while a high-irradiance protocol resulted in riboflavin consumption faster than conventional irradiance when the stroma was fully enriched with the therapeutic molecule [30]. Pulsed UVA illumination can achieve higher singlet oxygen concentrations for crosslinking of collagen molecules. According to photochemical crosslinking studies based on a kinetics model, UVA illumination in a riboflavin-soaked cornea causes a rapid depletion of the oxygen. On the other hand, turning the UV light off leads to replenishment of the oxygen to its original levels within 3-4 min. Pulsing the UV light theoretically increases the oxygen concentration by slowing the rate of oxygen consumption and restarts the photodynamic type II reaction achieving an additional oxygen concentration for crosslinking of collagen molecules [8]. El Hout et al. [31] introduced a custom-made oxygen delivery mask connected to an oxygen wall outlet with a flow meter and bubble humidifier in order to achieve higher oxygen concentrations in the cornea with promising results. 
Oxygen is an important element in the collagen-CXL reaction [8]. The presence of oxygen together with sufficient riboflavin penetration and adequate UVA exposure are required for effective crosslinking treatment [29]. The use of pulsed-light compared to continuous light may improve availability of oxygen during CXL treatment. Considering the riboflavin concentration routinely used, there is adequate evidence in the literature about the efficacy of pulsed accelerated treatments. Different chromophores or different riboflavin concentrations could be investigated in the future. Their potential interaction with various irradiation energy doses may enhance tissue effects and modify our current protocols.

\section{Conclusion}

In conclusion, corneal stroma demarcation line depth did not differ significantly between our pulsed, accelerated CXL protocol and the Dresden protocol. These two techniques may have similar efficacies in the treatment of KC.

\section{Acknowledgements}

We thank Goula Elisavet (MD) for editing the language.

\section{Statement of Ethics}

The study was approved by the Ophthalmica Eye Institute Ethical Committee in accordance with the ethical standards laid down in the Declaration of Helsinki (seventh revision, October 2013, Fortaleza, Brazil). The study participants provided informed consent.

\section{Disclosure Statement}

The authors have no conflicts of interest to declare.

\section{Funding Sources}

The authors did not receive any funding for this study.

\section{Author Contributions}

N.D. performed the statistical analysis. All authors participated in study design, read, and approved the final manuscript.

\section{References}

1 Rabinowitz YS. Keratoconus. Surv Ophthalmol. 1998 Jan-Feb;42(4):297-319.

2 Ertan A, Colin J. Intracorneal rings for keratoconus and keratectasia. J Cataract Refract Surg. 2007 Jul;33(7):1303-14.

3 Ziaei M, Barsam A, Shamie N, Vroman D, Kim T, Donnenfeld ED, et al.; ASCRS Cornea Clinical Committee. Reshaping procedures for the surgical management of corneal ectasia. J Cataract Refract Surg. 2015 Apr;41(4): 842-72.

4 Raiskup-Wolf F, Hoyer A, Spoerl E, Pillunat LE. Collagen crosslinking with riboflavin and ultraviolet-A light in keratoconus: long-term results. J Cataract Refract Surg. 2008 May; 34(5):796-801.

5 O'Brart DP, Kwong TQ, Patel P, McDonald RJ, O'Brart NA. Long-term follow-up of riboflavin/ultraviolet A $(370 \mathrm{~nm})$ corneal collagen cross-linking to halt the progression of keratoconus. Br J Ophthalmol. 2013 Apr;97(4): 433-7.

6 Wollensak G, Spoerl E, Seiler T. Riboflavin/ ultraviolet-a-induced collagen crosslinking for the treatment of keratoconus. Am J Ophthalmol. 2003 May;135(5):620-7.

7 Brindley GS. The Bunsen-Roscoe law for the human eye at very short durations. J Physiol. 1952 Sep;118(1):135-9.
8 Kamaev P, Friedman MD, Sherr E, Muller D. Photochemical kinetics of corneal cross-linking with riboflavin. Invest Ophthalmol Vis Sci. 2012 Apr;53(4):2360-7.

9 Kymionis GD, Portaliou DM, Kounis GA, Limnopoulou AN, Kontadakis GA, Grentzelos MA. Simultaneous topography-guided photorefractive keratectomy followed by corneal collagen cross-linking for keratoconus. Am J Ophthalmol. 2011 Nov;152(5):748-55.

10 Kanellopoulos AJ, Asimellis G. Keratoconus management: long-term stability of topography-guided normalization combined with high-fluence CXL stabilization (the Athens Protocol). J Refract Surg. 2014 Feb;30(2):8893.

11 Seiler T, Hafezi F. Corneal cross-linkinginduced stromal demarcation line. Cornea. 2006 Oct;25(9):1057-9.

12 Doors M, Tahzib NG, Eggink FA, Berendschot TT, Webers CA, Nuijts RM. Use of anterior segment optical coherence tomography to study corneal changes after collagen crosslinking. Am J Ophthalmol. 2009;148:844-51. e2.

13 Kymionis GD, Grentzelos MA, Plaka AD, Tsoulnaras KI, Diakonis VF, Liakopoulos DA, et al. Correlation of the corneal collagen cross-linking demarcation line using confocal microscopy and anterior segment optical coherence tomography in keratoconic patients. Am J Ophthalmol. 2014;157:110-15.e1.

14 Mazzotta C, Traversi C, Caragiuli S, Rechichi M. Pulsed vs continuous light accelerated corneal collagen crosslinking: in vivo qualitative investigation by confocal microscopy and corneal OCT. Eye (Lond). 2014 Oct;28(10): 1179-83.

15 Pircher N, Lammer J, Holzer S, Gschließer A, Donner R, Pieh S, et al. Correlation between central stromal demarcation line depth and changes in $\mathrm{K}$ values after corneal cross-linking (CXL). Graefes Arch Clin Exp Ophthalmol. 2018 Apr;256(4):759-64.

16 Gatzioufas Z, Richoz O, Brugnoli E, Hafezi F. Safety profile of high-fluence corneal collagen cross-linking for progressive keratoconus: preliminary results from a prospective cohort study. J Refract Surg. 2013 Dec;29(12):846-8.

17 Hashemian H, Jabbarvand M, Khodaparast M, Ameli K. Evaluation of corneal changes after conventional versus accelerated corneal cross-linking: a randomized controlled trial. J Refract Surg. 2014 Dec;30(12):837-42.

18 Konstantopoulos A, Mehta JS. Conventional versus accelerated collagen cross-linking for keratoconus. Eye Contact Lens. 2015 Mar; $41(2): 65-71$. 
19 Shetty R, Pahuja NK, Nuijts RM, Ajani A, Jayadev C, Sharma C, et al. Current protocols of corneal collagen cross-linking: visual, refractive, and tomographic outcomes. Am J Ophthalmol. 2015 Aug;160(2):243-9.

20 Elbaz U, Shen C, Lichtinger A, Zauberman NA, Goldich Y, Chan CC, et al. Accelerated $(9-\mathrm{mW} / \mathrm{cm} 2)$ corneal collagen crosslinking for keratoconus-A 1-year follow-up. Cornea. 2014 Aug;33(8):769-73.

21 Marino GK, Torricelli AA, Giacomin N, Santhiago MR, Espindola R, Netto MV. Accelerated corneal collagen cross-linking for postoperative LASIK ectasia: two-year outcomes. J Refract Surg. 2015 Jun;31(6):380-4.

22 Hammer A, Richoz O, Arba Mosquera S, Tabibian D, Hoogewoud F, Hafezi F. Corneal biomechanical properties at different corneal cross-linking (CXL) irradiances. Invest Ophthalmol Vis Sci. 2014 May;55(5):2881-4.

23 Kymionis GD, Tsoulnaras KI, Grentzelos MA, Plaka AD, Mikropoulos DG, Liakopoulos DA, et al. Corneal stroma demarcation line after standard and high-intensity collagen crosslinking determined with anterior segment optical coherence tomography. J Cataract Refract Surg. 2014 May;40(5):73640.

24 Mita M, Waring GO 4th, Tomita M. Highirradiance accelerated collagen crosslinking for the treatment of keratoconus: six-month results. J Cataract Refract Surg. 2014 Jun; 40(6):1032-40.

25 Hashemi H, Fotouhi A, Miraftab M, Bahrmandy $\mathrm{H}$, Seyedian MA, Amanzadeh K, et al. Short-term comparison of accelerated and standard methods of corneal collagen crosslinking. J Cataract Refract Surg. 2015 Mar; 41(3):533-40

26 Bowes O, Coutts S, Ismailjee A, Trocme E, Vilella AJ, Perry H, et al. Pulsed light accelerated corneal collagen cross-linking: 1-year results. Cornea. 2017 Jun;36(6):e15-6.

27 Shajari M, Sowwa G, Steinwender G, Hofmann C, Pahlitzsch ML, Petermann K, et al. Comparison of Corneal Collagen Cross-Link- ing Protocols Measured With Scheimpflug Tomography. Cornea. 2018 Jul;37(7):870-4.

28 Mazzotta C, Hafezi F, Kymionis G, Caragiuli S, Jacob S, Traversi C, et al. In vivo confocal microscopy after corneal collagen crosslinking. Ocul Surf. 2015 Oct;13(4):298-314.

29 Krueger RR, Herekar S, Spoerl E. First proposed efficacy study of high versus standard irradiance and fractionated riboflavin/ultraviolet a cross-linking with equivalent energy exposure. Eye Contact Lens. 2014 Nov;40(6): 353-7.

30 Lombardo M, Lombardo G. Noninvasive real-time assessment of riboflavin consumption in standard and accelerated corneal crosslinking. J Cataract Refract Surg. 2019 Jan;45(1): 80-6.

31 El Hout S, Cassagne M, Sales de Gauzy T, Galiacy S, Malecaze F, Fournié P. Transepithelial photorefractive intrastromal corneal crosslinking versus photorefractive keratectomy in low myopia. J Cataract Refract Surg. 2019 Apr;45(4):427-36. 